\title{
The Role of Suboptimal Concentrations of Nebulized Tobramycin in Driving Antimicrobial Resistance in Pseudomonas aeruginosa Isolates in Cystic Fibrosis
}

\author{
John E Moore, B Cherie Millar, Marika Ollman-Selinger, and Lisa Cambridge
}

\begin{abstract}
BACKGROUND: Antimicrobial resistance in Pseudomonas aeruginosa may be driven by exposure to suboptimal concentrations of tobramycin antibiotic delivered by less efficient nebulizers. METHODS: $P$. aeruginosa isolates (no. $=114 ; 32$ first +82 chronic) were challenged in vitro employing extrapolated peak and trough concentrations of tobramycin inhalation solution (TIS), corresponding to 3 nebulizers: Pari LC Plus, Sidestream12NEB400, and MistyNeb2035G. Bacterial persistence and antibiotic susceptibility to tobramycin was determined following 4 TIS cycles: (i) $28 \mathrm{~d}$ ON, (ii) $28 \mathrm{~d}$ ON $+28 \mathrm{~d}$ OFF, (iii) $2 \times 28 \mathrm{~d}$ ON, and (iv) $28 \mathrm{~d} \mathrm{ON}+28 \mathrm{~d} \mathrm{ON}+$ 28 d OFF. RESULTS: All first isolates were eradicated at peak and trough concentrations except for the trough concentration corresponding to Sidestream 12NEB400 (bactericidal activity 87\%). For chronic isolates, peak concentrations eradicated $88 \%, 90 \%$, and $92 \%$, and trough concentrations eradicated 43\%, 62\%, and 85\%, with the Sidestream12NEB400, MistyNeb2035G, and Pari LC Plus nebulizers, respectively. A statistically significant increase in antibiotic resistance with sensitive, intermediate, and resistant $\boldsymbol{P}$. aeruginosa was noted following cycles (i) through (iv) at trough concentrations with the Sidestream 12NEB400 and MistyNeb2035G nebulizers. There was a significant reduction in tobramycin resistance following a 28-d OFF cycle, and no difference was noted following $1 \times 28 \mathrm{~d}$ ON versus $2 \times 28 \mathrm{~d}$ ON cycles. CONCLUSIONS: Our results indicate that suboptimal concentrations of tobramycin drove increased antibiotic resistance, emulating standard cycles of $\mathrm{ON} / \mathrm{OFF}$ inhaled therapy. This was evident at extrapolated tobramycin concentrations at trough levels corresponding to less efficient nebulizers by initially allowing for the survival of intermediate and resistant organisms, because nebulizer performance did not achieve critical antibiotic concentrations sufficient to eradicate the organism, and by allowing the development of resistance in those cells that were able to survive the initial tobramycin challenge. Transferred to clinical practice, for people with cystic fibrosis on TIS treatment, it is important that clinicians employ an efficient nebulizer that helps mitigate an upward drift in antibiotic resistance, thereby protecting the clinical value of TIS within treatment for cystic fibrosis. Key words: Pseudomonas aeruginosa; tobramycin inhaled solution; nebulizer performance; antimicrobial resistance; bacterial persister; cystic fibrosis antibiotic cycling. [Respir Care 2021;66(9):1446-1457. (C) 2021 Daedalus Enterprises]
\end{abstract}

\section{Introduction}

Cystic fibrosis $(\mathrm{CF})$ is the most inherited autosomal recessive lethal genetic disease in the white population, accounting for approximately 70,000 people with CF globally and approximately 30,000 persons within the United States. ${ }^{1}$ The basis for the disease is genetic mutations within the cystic fibrosis transmembrane conductance regulator (CFTR) protein, leading to absence or abnormalities of this protein, which results in problems in the cells' ability to transport chloride ions across cell membranes. This results in sticky mucus that blocks the pancreatic duct and the gastrointestinal tract and alters airway clearance and antimicrobial defenses within the lungs. Failure to clear mucus results in the entrapment of many environmental bacteria and fungi, particularly the bacterial pathogen Pseudomonas aeruginosa. This leads to a vicious cycle of infection and inflammation, ultimately resulting in 
increased morbidity and mortality. Therefore any treatments (eg, oral, nebulized, intravenous antibiotics) and therapies (eg, mucolytic and airway clearance techniques) that help eliminate $P$. aeruginosa from the airways of individuals with $\mathrm{CF}$ will help reduce the microbiological burden of this organism, suppress pulmonary exacerbations, minimize respiratory inflammation, and slow any decline in lung function.

The Cystic Fibrosis Foundation recommends inhaled tobramycin in mild and in moderate-to-severe lung disease, on the basis of evidence from 3 randomized controlled trials involving 234 subjects and 1 randomized crossover trial involving 1,110 subjects who substantially benefited from treatment. ${ }^{2}$ Several other international CF Standards of Care Guidelines advocate tobramycin for maintenance therapy in chronic infection, as well as for eradication therapy on first isolation of $P$. aeruginosa. ${ }^{3,4}$ Inhaled tobramycin is available in 2 formulations, either as a tobramycin inhalation solution (TIS) or as a dry powder. The dry powder tobramycin formulation is commonly used, but it is limited to those who can properly demonstrate the technique. Two Cochrane reviews support the use of inhaled tobramycin as an antipseudomonal antibiotic for both eradication and maintenance therapy. ${ }^{3,5}$

To be clinically effective at killing $P$. aeruginosa, adequate concentrations of nebulized tobramycin must be delivered to the CF airways. More specifically, these concentrations need to be above the threshold antibiotic concentration, known as the minimum inhibitory concentration (MIC) of the new or chronically colonizing $P$. aeruginosa strain. Several factors can be responsible for the occurrence of sublethal tobramycin concentrations (ie, below the MIC) within the CF airway, as detailed in Table

Drs Moore and Millar are affiliated with the Northern Ireland Public Health Laboratory, Belfast City Hospital, Belfast, Northern Ireland, United Kingdom. Dr Ollman-Selinger is affiliated with Pari $\mathrm{GmbH}$, Starnberg, Germany. Ms Cambridge is affiliated with Pari Respiratory Equipment, Midlothian, Virginia.

Dr Moore presented a version of this paper at the 2020 North American Cystic Fibrosis Conference, held virtually October 21-23, 2020.

Supplementary material related to this paper is available at http://www. rcjournal.com.

This study was funded by Pari Respiratory Equipment. Drs Moore and Millar have disclosed relationships with Pari Respiratory Equipment. Dr Ollman-Selinger is an employee of Pari GmbH, and Ms Cambridge is an employee of Pari Respiratory Equipment.

Correspondence: John E Moore PhD, Northern Ireland Public Health Laboratory, Department of Bacteriology, Belfast City Hospital, Belfast BT9 7AD, Northern Ireland, United Kingdom. E-mail: jemoore@niphl.dnet.co.uk.

DOI: $10.4187 /$ respcare.08671

\section{QUICK LOOK}

\section{Current knowledge}

Nebulized tobramycin is a first-line therapy for the eradication and chronic suppression of the bacterial pathogen $P$. aeruginosa in people with cystic fibrosis. Tobramycin is mainly delivered to the lungs via jet nebulizers that have varied performance characteristics. These differences in delivery efficiencies are based on aerosol characteristics such as respirable dose, which is the amount of drug that can reach the lower airway, mass median aerodynamic diameter, fine particle fraction $(\%<5 \mu \mathrm{m})$, and fine particle mass.

\section{What this paper contributes to our knowledge}

Concentrations of tobramycin inhalation solution below the minimum inhibitory concentration, emulating inefficient nebulizer delivery, drove bacterial persistence and antibiotic resistance with $P$. aeruginosa in vitro. In vitro cycling of $28 \mathrm{~d}$ on and $28 \mathrm{~d}$ off of tobramycin inhalation solution improved $P$. aeruginosa susceptibility to tobramycin. Efficient nebulizer choice is important to minimize bacterial persistence and tobramycin antibiotic resistance development.

$1 .^{8,9}$ Unlike patient-related factors, such as adherence to nebulized tobramycin therapy or disease characteristics, device choice is an important aspect of CF therapy that clinicians can control. Clinicians can optimize delivery of TIS by selecting an efficient nebulizer based on published performance data. ${ }^{10}$

Several studies have examined the effects of nebulizer efficiency on tobramycin delivery to the CF lungs. ${ }^{6,11-15}$ In a related Cochrane review investigating nebulizer systems for drug delivery in cystic fibrosis, the authors concluded that there was variability in the performance of different nebulizer systems. ${ }^{5}$

In the United Kingdom, nebulized tobramycin is approved specifically with the Pari LC Plus nebulizer (Pari GmbH, Starnberg, Germany). ${ }^{7}$ In the United States, since the seminal paper by Ramsey et al ${ }^{16}$ the Food and Drug Administration has historically specified that TIS should be administered via a Pari LC Plus nebulizer. Presently, there are 10 commercially available nebulized tobramycin formulations listed in the FDA's Orange Book (40th Edition), in addition to the United States Pharmacopeia. All of these compendia specify that TIS should be delivered via a Pari LC Plus nebulizer. ${ }^{17}$ While this is the case, health care providers and individuals with CF may opt to deliver nebulized tobramycin via an 


\section{Nebulized Tobramycin Concentration and Antimicrobial Resistance}

Table 1. Factors Responsible for Suboptimal Concentration of Tobramycin in Cystic Fibrosis Airways

\begin{tabular}{l} 
Tobramycin concentrations in the airways are highly variable for a \\
variety of reasons: \\
Patient-related factors \\
Breathing pattern \\
Airway anatomy \\
Age \\
Sputum production/volume \\
Airway obstruction \\
Degree of mucus plugging \\
Disease severity \\
Patient adherence to nebulized tobramycin therapy \\
Previous adherence to nebulizer therapy, as a percentage of the \\
prescribed regimen, has been reported as $65-80 \%$ via self- \\
reporting, 50-60\% via clinician reporting, and 36\% via electronic \\
download \\
The inhibitory effect of sputum constituents that bind aminoglycosides, \\
including \\
Mucin \\
Glycoprotein \\
Free eukaryotic and bacterial DNA \\
Biofilm age, as older biofilms are composed of more resistant bacteria \\
(100-1,000-fold greater) compared to younger biofilms \\
Device-related factors including nebulizer type and efficiency \\
\hline
\end{tabular}

alternative nebulizer because individuals may not always have access to the Pari LC Plus. TIS is a common therapy in patients with chronic $P$. aeruginosa and is the third most common medication prescription after dornase alfa and hypertonic saline. ${ }^{18}$ In $2018,70.2 \%$ of people with $\mathrm{CF}$ with $P$. aeruginosa ( $\geq 6 \mathrm{y}$ of age) were taking inhaled tobramycin. In children, inhaled tobramycin was used therapeutically in $18.5 \%$ ( $<3 \mathrm{y}$ of age, $n=1,877$ ) and in $22.1 \%$ of children age $3-5 \mathrm{y}(n=2,272) .{ }^{18}$ A recent U.S. survey conducted among $\mathrm{CF}$ patients and providers indicated that, among the $671 \mathrm{CF}$ community responses from 48 states, Pari brand devices were the most commonly used $(50 \%) .{ }^{19}$ The survey also showed that $46 \%$ of responders did not have enough nebulizer cups. Given these data and high utilization $(70.2 \%$ of those with $P$. aerugi$n o s a$ ) of inhaled tobramycin, it is anticipated that there are individuals who are using nebulizers other than the Pari LC Plus to deliver TIS. A previous study that compared the efficiency of 18 disposable nebulizer systems and 2 reusable nebulizer systems indicated that none of the disposable nebulizers tested could be recommended as an alternative to the Pari LC Plus nebulizer for tobramycin delivery due to lower lung deposition (see the supplementary materials at http://www.rcjournal.com). ${ }^{10}$

Antimicrobial resistance is an emerging public health problem globally; however, it is a particularly significant problem in chronically infecting organisms of the airways of individuals with $\mathrm{CF} .{ }^{20}$ In the United States, rates of multidrug-resistant $P$. aeruginosa infection are greatest in older adolescents and adults with $\mathrm{CF}$, probably reflecting cumulative exposure to antibiotics. Among those people with $\mathrm{CF}$ who had at least one bacterial culture in 2018, 7.5\% were reported to have multidrug-resistant $P$. aeruginosa.$^{18}$ Additionally, among people with CF with $P$. aeruginosa infection in 2018, 16.9\% were reported to have multidrug-resistant $P$. aeruginosa.$^{18}$ However, the Cystic Fibrosis Foundation Registry reports that the clinical importance of such antibiotic resistance remains unclear. Elsewhere, increasing antibiotic resistance to tobramycin has been reported. ${ }^{21}$ In Northern Ireland, a recent study reported that tobramycin resistance increased significantly $(P<.001)$ over the period of 1996 to $2016 .^{22}$

The importance of inhaled antibiotics in CF, in terms of the clinical benefits derived from such therapies, has been well established, with extensive information supporting its safety and efficacy. ${ }^{23}$ Because inhaled tobramycin is considered a first-line antipseudomonal treatment, it has been suggested that high local concentrations delivered by this route may help overcome resistance. ${ }^{24-26}$ It has been reported that exposure of suboptimal concentrations of antibiotics may lead to acquisition of antimicrobial resistance. ${ }^{27}$ We therefore hypothesized that suboptimal delivery of lower concentrations of tobramycin to the lungs by less efficient nebulizers may have an effect on the antibiotic susceptibility of $P$. aeruginosa.

Therefore, the aims of this study were to examine: the in vitro survival of $P$. aeruginosa when challenged with extrapolated peak/trough concentrations of tobramycin emulating delivery by 3 nebulizer devices used in $\mathrm{CF}$ nebulized therapy; the in vitro drift of tobramycin susceptibility in $P$. aeruginosa when challenged with tobramycin emulating peak/trough nebulizer extrapolated concentrations with 4 treatment regimen combinations; and the in vitro effect of a 28-d OFF period on $P$. aeruginosa tobramycin susceptibility.

\section{Methods}

A series of in vitro experiments were designed to emulate the delivery of nebulized tobramycin. A summary of the microbiological procedures employed in this study is shown in Figure 1.

\section{Description of $\boldsymbol{P}$. aeruginosa Isolates}

This was entirely an in vitro study and did not involve the collection of specimens from any CF individual for the purposes of this study. All isolates employed in this study were obtained from an existing public bacterial isolate repository, the HSC Microbiology Isolate Culture Collection 


\section{Methods}

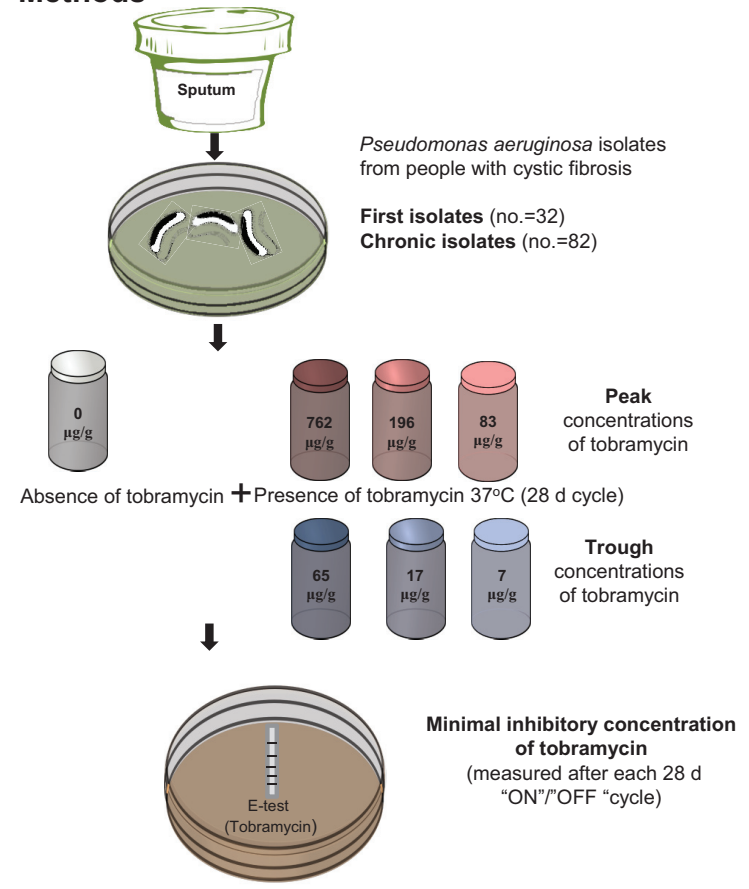

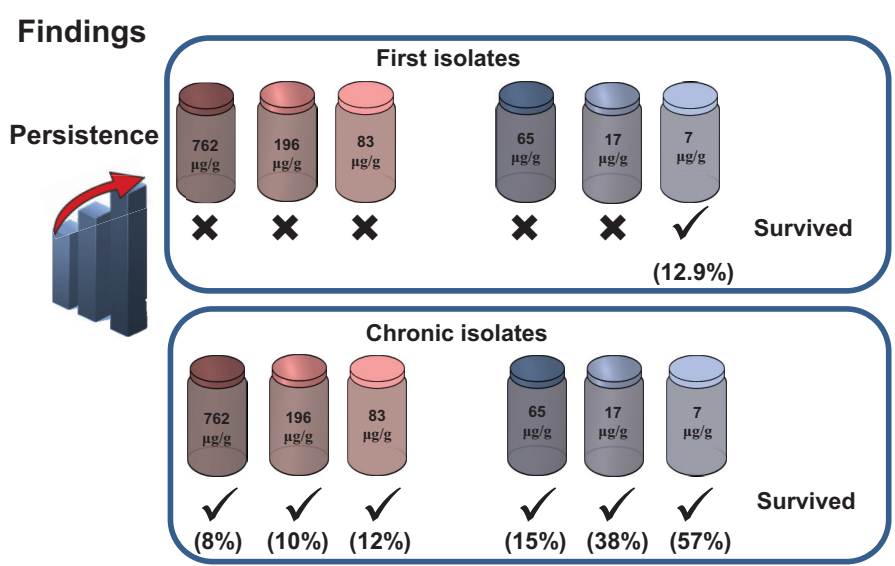

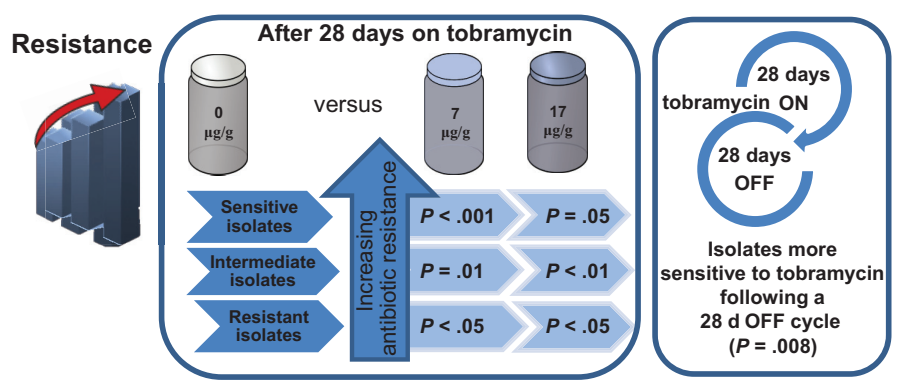

Take Home Messages

It is important that both clinicians advocate and that patients use the nebulizer device which maximizes tobramycin delivery and cycle antibiotic use to: (i) optimise the eradication of first isolates and (ii) help mitigate the development of antibiotic resistance, particularly during chronic infection.

Fig. 1. Concentrations of tobramycin below the minimum inhibitory concentration are a driver of persistence and antibiotic resistance in $P$. aeruginosa isolates from individuals with cystic fibrosis.

Repository (CF MicroARK) housed at the Northern Ireland Public Health Laboratory. No extra or additional requests for sputum were made from individuals with $\mathrm{CF}$. Because all $P$. aeruginosa isolates employed in this study were completely anonymous, no clinical patient identifiers or other information were available.

All experiments were performed at the Northern Ireland Public Health Laboratory, Belfast City Hospital, Belfast, United Kingdom. The identification of each isolate was confirmed with analysis using matrix-assisted laser desorption/ionization/time-of-flight mass spectrometry. Prior to use, a purified single colony of each isolate was passaged 3 times by subculturing on Columbia Blood agar (Oxoid CM0031, Oxoid, Basingstoke, United Kingdom), supplemented with $5 \%(\mathrm{v} / \mathrm{v})$ defibrinated horse blood for $24 \mathrm{~h}$ at $37^{\circ} \mathrm{C}$ under aerobic conditions. In addition, the reference strain, $P$. aeruginosa WDCM000025 (ATCC 27853), was used as a control.

\section{Peak/Trough Tobramycin Concentrations Used}

As this was an in vitro study, nebulizers were not physically employed as such; therefore, peak and trough tobramycin concentrations are subsequently referred to previously published information (Pari LC Plus) or extrapol- ated (Sidestream 12NEB400 and MistyNeb2035G). The tobramycin concentrations used in this study are shown in Table 2. In the case of the FDA-specified nebulizer for TIS delivery (ie, the Pari LC Plus), the concentrations used were obtained from a pharmacokinetic safety study by Hubert et $\mathrm{al},{ }^{14}$ where sputum and serum concentration deposition of TIS $(300 \mathrm{mg} / 5 \mathrm{~mL}$ ) delivered with the Pari LC Plus nebulizer with a Pulmo-Aide compressor (DeVilbiss, Somerset, Pennsylvania) administered twice daily over $15 \mathrm{~d}$ was investigated in individuals with $\mathrm{CF}$ with chronic $P$. aeruginosa infection. ${ }^{14}$ In the case of 2 less efficient nebulizers commonly used in the United States, as reported by Vecellio et $\mathrm{al}^{10}$ (based on the fine particle mass being $>20 \%$ lower), namely the Sidestream 12NEB400/wall air (Respironics, Murrysville, Pennsylvania) and the MistyNeb2035G/PulmoAide compressor (Cardinal Health, Dublin, Ohio), extrapolated tobramycin concentrations were determined as shown in the supplementary materials (available at http://www. rcjournal.com).

\section{Description of Tobramycin Cycling Regimens}

Four tobramycin cycling regimens (arms 1-4) were identified and adopted into the study protocol as detailed in Figure 


\section{Nebulized Tobramycin Concentration and Antimicrobial Resistance}

Table 2. Tobramycin Concentrations Employed in This Study

\begin{tabular}{lccc}
\hline \hline \multicolumn{1}{c}{$\begin{array}{c}\text { Peak } \\
\text { Nebulizer }\end{array}$} & $\begin{array}{c}\text { Trough } \\
\text { Tobramycin } \\
\text { Concentration, } \\
\mu \mathrm{g} / \mathrm{g}\end{array}$ & $\begin{array}{c}\text { Tobramycin } \\
\text { Concentration, } \\
\mu \mathrm{g} / \mathrm{g}\end{array}$ & $\begin{array}{c}\text { Fine } \\
\text { Particle } \\
\text { Mass, mg }\end{array}$ \\
\hline Pari LC Plus & $762^{*}$ & $65^{*}$ & $74 \pm 6$ \\
Misty Neb 2035G & $196^{\dagger}$ & $17^{\dagger}$ & $19 \pm 2$ \\
Sidestream 12-NEB400 & $83^{\dagger}$ & $7^{\dagger}$ & $8 \pm 7$ \\
& & & \\
${ }^{*}$ From Hubert et al ${ }^{14}$ (peak tobramycin concentration ranged from 754 to $\left.769 \mu \mathrm{g} / \mathrm{g}\right)$. \\
${ }^{\dagger}$ Extrapolated from Vecellio et al. ${ }^{10}$ \\
\end{tabular}

\begin{tabular}{|c|c|c|c|}
\hline ARM 1 & ARM 2 & ARM 3 & ARM 4 \\
\hline $\begin{array}{c}28 \text { days } \\
\text { ON }\end{array}$ & $\begin{array}{c}28 \text { days } \\
\text { ON } \\
+\end{array}$ & $\begin{array}{c}28 \text { days } \\
\text { ON } \\
+\end{array}$ & $\begin{array}{c}28 \text { days } \\
\text { ON } \\
+\end{array}$ \\
\hline & $\begin{array}{c}28 \text { days } \\
\text { OFF }\end{array}$ & $\begin{array}{c}28 \text { days } \\
\text { ON }\end{array}$ & $\begin{array}{c}28 \text { days } \\
\text { ON } \\
+\end{array}$ \\
\cline { 2 - 5 } & \multicolumn{2}{|c}{$\begin{array}{c}28 \text { days } \\
\text { OFF }\end{array}$} \\
\hline
\end{tabular}

Fig. 2. Description of in vitro tobramycin cycling regimens used in this study.

2. An initial pilot study was undertaken with $P$. aeruginosa (no. $=20$ isolates) to ascertain whether $P$. aeruginosa was able to survive incubation in nutrient broth (Oxoid CM1) for $56 \mathrm{~d}(2 \times 28 \mathrm{~d}$ cycles). When switching from an ON cycle (ie, in the presence of tobramycin) to an OFF cycle, an inoculum $(10 \mu \mathrm{L})$ of incubated culture from the previous ON cycle with tobramycin was subcultured into fresh nutrient broth $(10 \mathrm{~mL})$ in the absence of tobramycin.

\section{Determination of Initial Tobramycin Susceptibility}

All $114 P$. aeruginosa clinical isolates, as well as the control P. aeruginosa WDCM000025 (ATCC ${ }^{\mathrm{TM}} 27853$ ), were initially screened for tobramycin susceptibility, using disk diffusion (10 $\mu \mathrm{g}$ tobramycin disk, Oxoid), in accordance with Clinical and Laboratory Standards Institute (CLSI) methodology and interpretive criteria, ${ }^{28}$ namely where zone size $\geq 15 \mathrm{~mm}=$ sensitive $(\mathrm{S}) ; 13-14 \mathrm{~mm}=$ intermediate (I), and $\leq 12 \mathrm{~mm}=$ resistant $(\mathrm{R})$. Isolates were subsequently categorized into S, I, or R groupings. ${ }^{28}$

\section{Determination of Survival of $\boldsymbol{P}$. aeruginos $a$ Isolates at Peak/Trough Concentrations of Tobramycin}

The concentrations of tobramycin as detailed in Table 2 were prepared using Mueller-Hinton Broth No. 2 (MHB2)
(Sigma -Aldrich, Gillingham, Dorset, United Kingdom), in glass universals $(7 \mathrm{~mL})$. To this, a fresh inoculum of each $P$. aeruginosa isolate was individually added to give an initial bacterial density, in accordance with CLSI methodology; the inoculated broth was incubated at $37^{\circ} \mathrm{C}$ for $24 \mathrm{~h}^{28}$ The broths were then examined visually to check for bacterial growth as manifested by cloudiness/turbidity of the broth, and then they were incubated for another $24 \mathrm{~h}$. Positive broths were incubated as per the study arm conditions (Fig. 2).

\section{Determination of Tobramycin Susceptibility in Study Arms 1-4}

$P$. aeruginosa ( $n=38$ isolates) consisting of 8 sensitive (S) isolates (first $P$. aeruginosa isolates) (CLSI $\mathrm{MIC}_{\mathrm{TOB}} \leq$ $4 \mathrm{mg} / \mathrm{L}$ ), 14 intermediate (I) isolates (CLSI MIC $\mathrm{TOB}_{\mathrm{TOB}}=8$ $\mathrm{mg} / \mathrm{L}$ ), and 16 resistant $(\mathrm{R})$ isolates $\left(\mathrm{CLSI} \mathrm{MIC}_{\mathrm{TOB}} \geq 16\right.$ $\mathrm{mg} / \mathrm{L}$ ) were used. Isolates were co-cultured in MHB2 at each of the 6 tobramycin concentrations described previously (Table 2), as well as in a control broth containing no tobramycin, in accordance with each of the study arms (Arm 1 to Arm 4). The MIC of each isolate at each tobramycin concentration in each study arm was determined in accordance with CLSI antimicrobial susceptibility test methodology, employing tobramycin E-test strips (MIC range $0.064-1,024 \mathrm{mg} / \mathrm{L}$ ) (Liofilchem SRL, Roseto degli Abruzzi, Italy) and used in accordance with the manufacturer's instructions. Following tobramycin challenge in accordance with the study arms, MHB2 $(10 \mu \mathrm{l})$ containing viable $P$. aeruginosa was subcultured in nutrient broth (10 $\mathrm{mL}$ Oxoid CM1) and further incubated at $37^{\circ} \mathrm{C}$, in accordance with the study arms (Fig. 2).

\section{Statistical Analyses}

The compliance of parameters to a normal distribution was assessed using the Shapiro-Wilk test. Subsequently the EDISON-WMW: Exact Dynamic Programming Solution of the Wilcoxon-Mann-Whitney test was applied to examine statistical significance. ${ }^{29}$ All statistical $(P)$ values are detailed on the individual graphs and figures.

\section{Results}

\section{Description of $\boldsymbol{P}$. aeruginosa Isolates and Survival With Extended Culture}

$P$. aeruginosa isolates (no. $=115)$ were used in this study, consisting of 32 first isolates, 82 chronic isolates, and the $P$. aeruginosa reference strain, WDCM00025 (ATCC 27853). Results from a pilot study to investigate whether $P$. aeruginosa isolates could survive long-term incubation indicated that they were able to survive in nutrient broth at 

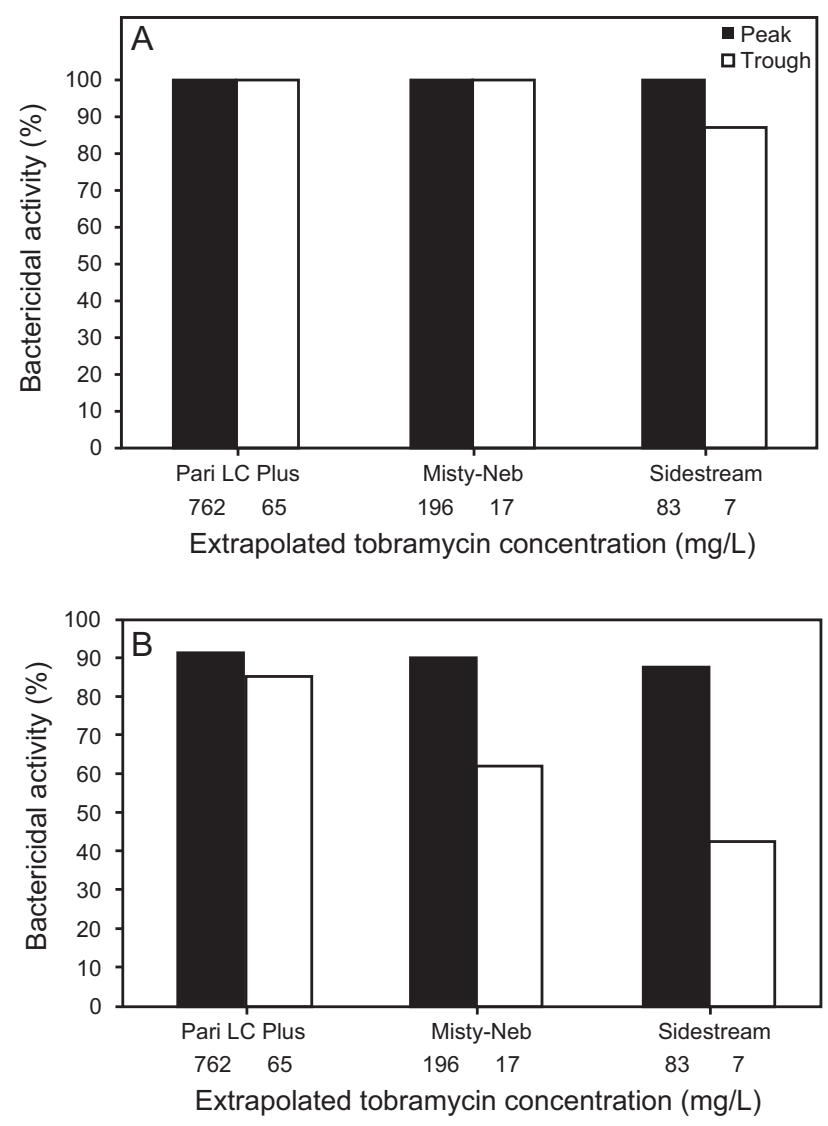

Fig. 3. A: Bactericidal activity (\%) of extrapolated nebulizer peak and trough tobramycin concentrations against first isolates of $P$. aeruginosa (no. = 32). B: Bactericidal activity (\%) of extrapolated nebulizer peak and trough tobramycin concentrations against chronic isolates of $P$. aeruginosa (no. $=82$ ).

$37^{\circ} \mathrm{C}$ for $\geq 56 \mathrm{~d}$ (see the supplementary materials at http:// www.rcjournal.com).

Our results indicate that peak and trough tobramycin concentrations from the Pari LC Plus and peak and trough concentrations with the MistyNeb2035G eradicated all first isolates (Fig. 3). However, while the extrapolated tobramycin peak concentration of the Sidestream nebulizer eradicated all organisms, its extrapolated trough concentration was unable to eradicate a small proportion of these isolates. When examining chronic isolates, none of the peak tobramycin concentrations from any of the nebulizers was able to eradicate all isolates, although there was a positive correlation with tobramycin concentration (see the supplementary materials at http://www.rcjournal.com). Likewise, there was wide variation in the ability of the extrapolated tobramycin concentrations to eradicate chronic isolates at their respective trough concentrations, ranging from $85 \%$ bactericidal activity for the Pari LC Plus to $43 \%$ bactericidal activity for the Sidestream (Fig. 3).

\section{Tobramycin Susceptibility in Study Arms 1-4}

$P$. aeruginosa isolates at the extreme ends of tobramycin susceptibility were exited from the study at this initial stage. Of the 114 original clinical isolates examined in this study, 62 were fully sensitive to the lowest concentration of tobramycin examined $(7 \mathrm{mg} / \mathrm{L})$ and were subsequently exited from the study. These isolates were exited at this stage because they would have been unable to survive the lowest concentration of tobramycin employed in subsequent study arms. A further 7 highly resistant isolates had an initial tobramycin sensitivity of $>1,024 \mathrm{mg} / \mathrm{L}$ and were also exited from the study because the method of tobramycin susceptibility testing employed (ie, quantitative E-test strips) had a maximum MIC value of $1,024 \mathrm{mg} / \mathrm{L}$, hence it would have been difficult to track changes in tobramycin susceptibility concentrations greater than the maximum value offered by the E-test strip. A total of 52 isolates were resistant to at least $7 \mathrm{mg} / \mathrm{L}$ tobramycin concentration; however, 14 of these isolates were excluded from the subsequent study arms due to nonsurvival of the control sample or contamination during the extended incubation durations of the study arms. A total of 38 P. aeruginosa isolates were subsequently followed through study arms 1-3.

Antibiotic susceptibility at each of the tobramycin coculture concentrations within arms 1-3 are shown in Figures 4-6, respectively. Figure 4 represents tobramycin susceptibility in $\mathrm{S}, \mathrm{I}$, and $\mathrm{R} P$. aeruginosa isolates (no. = 38) after $28 \mathrm{~d}$ on tobramycin (ie, ON). Figure 5 represents tobramycin susceptibility in $\mathrm{S}, \mathrm{I}$, and $\mathrm{R} P$. aeruginosa isolates (no. $=38$ ) after $28 \mathrm{~d} \mathrm{ON}+28 \mathrm{~d}$ OFF. Figure 6 represents tobramycin susceptibility in $\mathrm{S}, \mathrm{I}$, and $\mathrm{R} P$. aeruginosa isolates $($ no. $=38)$ after $2 \times 28 \mathrm{~d} \mathrm{ON}$ tobramycin.

In the case of isolates that remained viable following incubation with subinhibitory concentrations of tobramycin, the antibiotic susceptibility to tobramycin decreased, resulting in statistically higher MIC values. This was consistent throughout all 4 arms of the study (Figs. 4-6; see the supplementary materials at http://www.rcjournal.com). Additionally, this was evident with all antibiotic susceptibility classification of isolates (S, I, and R). The increase in MIC, although observed within all such classifications, was most notable in the sensitive and intermediate classes (Figs. 4-6; see the supplementary materials at http://www.rcjournal.com). Supplementary Figure 4 represents antibiotic susceptibility of total $P$. aeruginosa isolates following $2 \times 28 \mathrm{~d} \mathrm{ON}+28 \mathrm{~d}$ OFF tobramycin (available at http://www.rcjournal.com).

The effect of incubation duration on tobramycin susceptibility in $40 P$. aeruginosa isolates in the absence of tobramycin showed a decrease in the tobramycin MIC, that is, increased susceptibility (see the supplementary materials at http://www.rcjournal.com). This is important to note in the context of this in vitro study because any increase in tobramycin resistance was a direct effect of tobramycin co-culture 

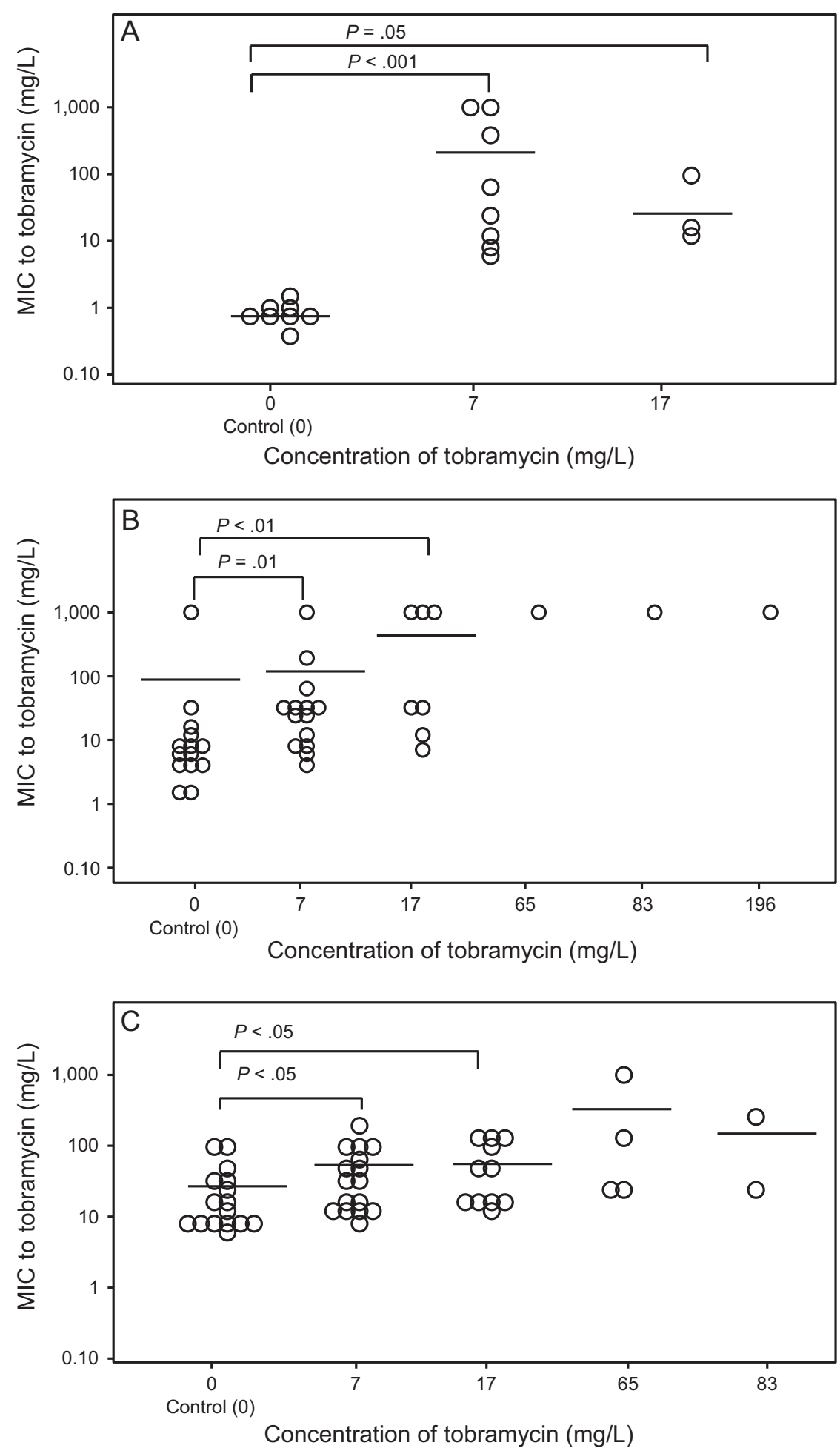

Fig. 4. A: Antibiotic susceptibility of sensitive isolates after $28 \mathrm{~d}$ on tobramycin. B: Antibiotic susceptibility of intermediate isolates after $28 \mathrm{~d}$ on tobramycin. C: Antibiotic susceptibility of resistant isolates after $28 \mathrm{~d}$ on tobramycin. $\mathrm{MIC}=$ minimum inhibitory concentration.

and not prolonged incubation. This effect may be attributed to a changing physiological environment of nutrient exhaustion, nutrient competition, accumulation of bacterial waste products, and metabolic shutdown/pseudodormancy, where the cells are preparing to enter a dormancy stage to remain viable and metabolically inactive. 

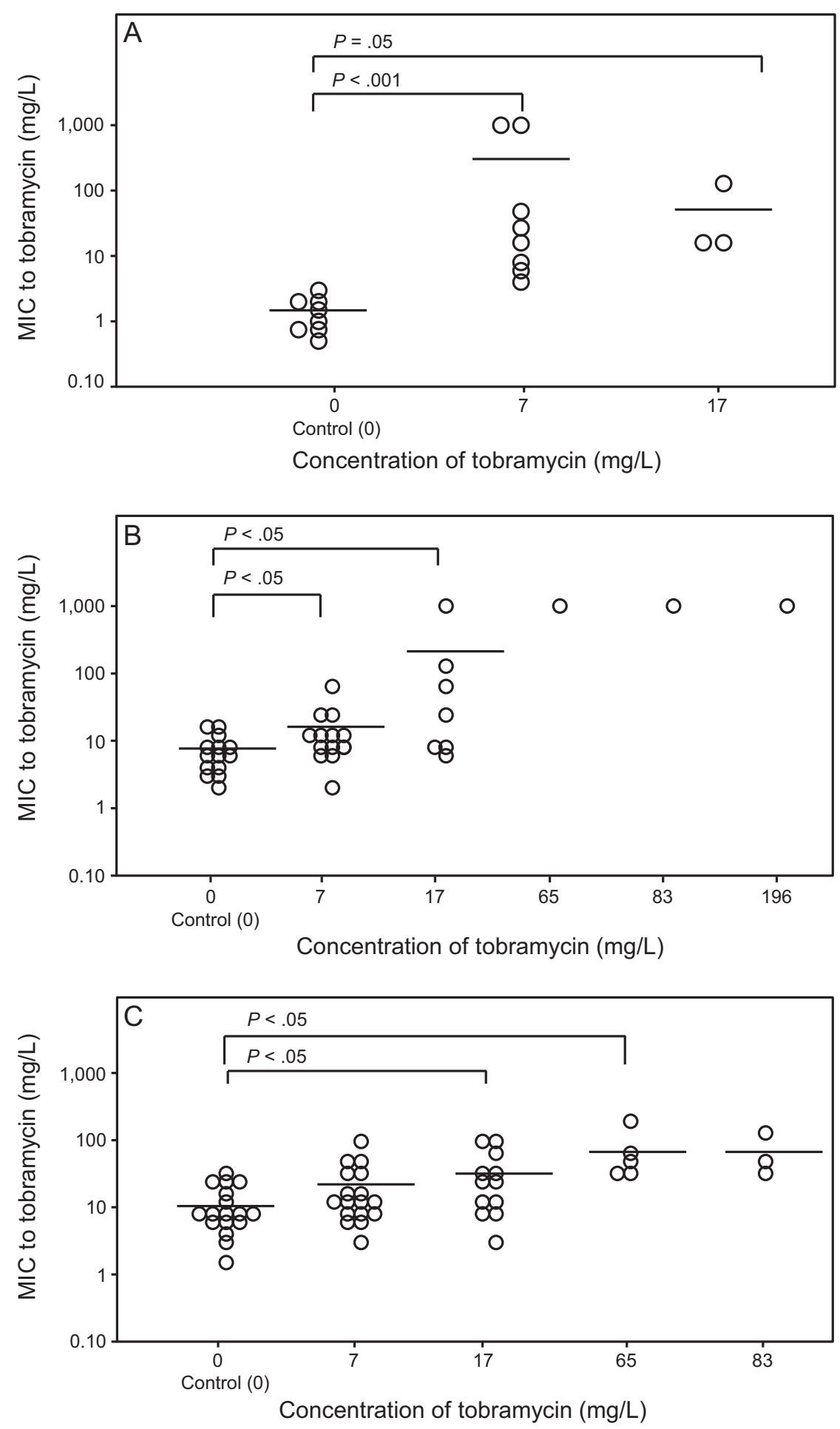

Fig. 5. A: Antibiotic susceptibility of sensitive isolates after $28 \mathrm{~d}$ on tobramycin and after $28 \mathrm{~d}$ off tobramycin. B: Antibiotic susceptibility of intermediate isolates after $28 \mathrm{~d}$ on tobramycin and after $28 \mathrm{~d}$ off tobramycin. C: Antibiotic susceptibility of resistant isolates after $28 \mathrm{~d}$ on tobramycin and after $28 \mathrm{~d}$ off tobramycin. MIC = minimum inhibitory concentration.

Supplementary Figure 6 (available at http://www.rcjournal. com) represents antibiotic susceptibility to tobramycin after incubation of $P$. aeruginosa isolates (no. $=38$ ) with tobramycin
(7 $\mathrm{mg} / \mathrm{L})$, in particular the effect of the 28-d OFF cycle on tobramycin susceptibility with $28 \mathrm{~d} \mathrm{ON}+28 \mathrm{~d}$ OFF versus $2 \times 28 \mathrm{~d}$ ON cycles. 

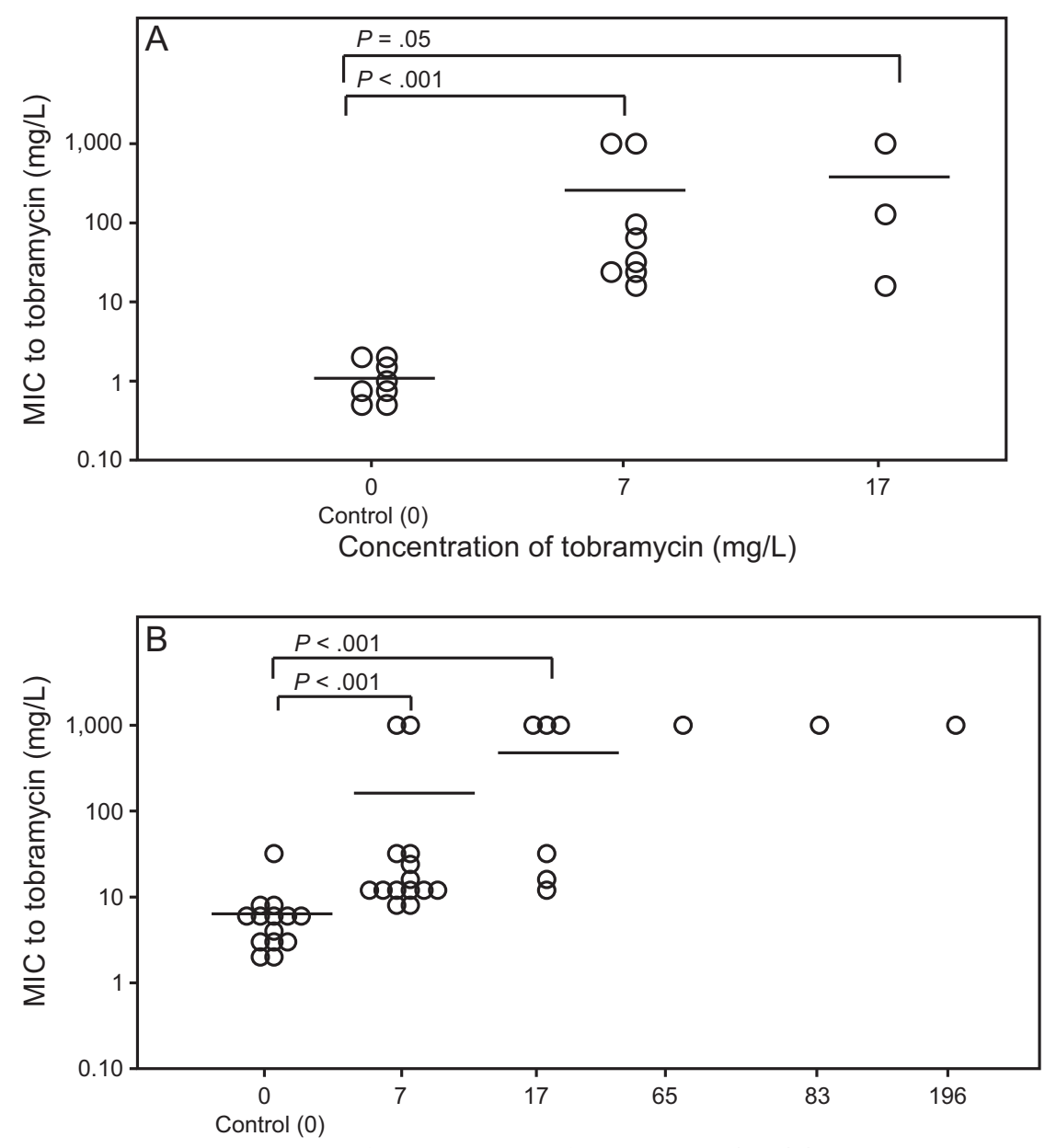

Concentration of tobramycin (mg/L)

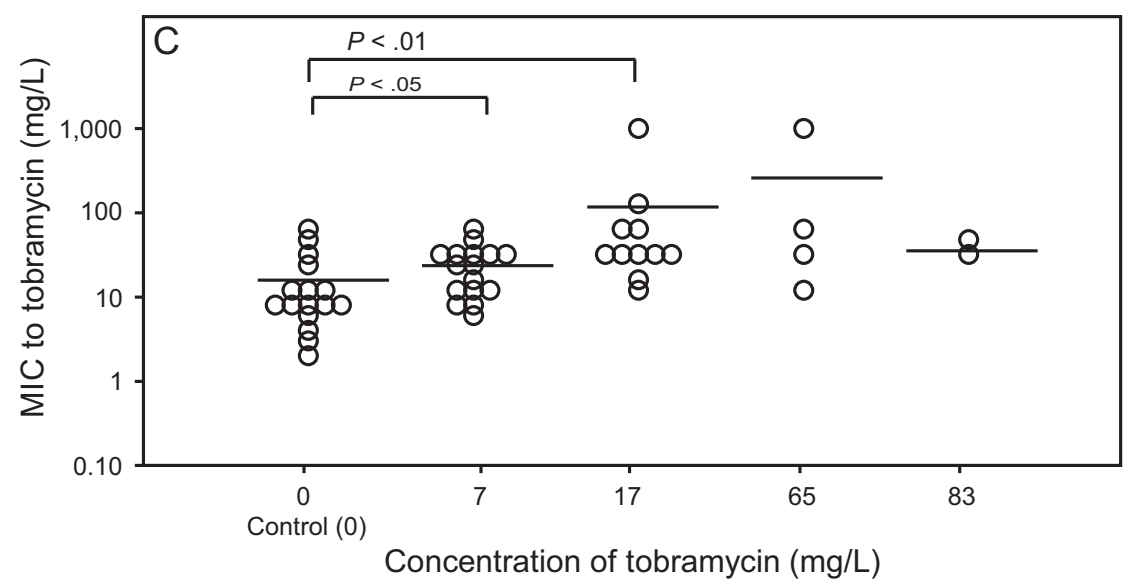

Fig. 6. A: Antibiotic susceptibility of sensitive isolates after $28 \mathrm{~d}$ on tobramycin $+28 \mathrm{~d}$ on tobramycin. B: Antibiotic susceptibility of intermediate isolates after $28 \mathrm{~d}$ on tobramycin $+28 \mathrm{~d}$ on tobramycin. C: Antibiotic susceptibility of resistant isolates after $28 \mathrm{~d}$ on tobramycin $+28 \mathrm{~d}$ on tobramycin. MIC = minimum inhibitory concentration.

\section{Discussion}

Less efficient nebulizers may be employed in the hospital setting as a cost-saving measure or for convenience, particularly relating to nebulizer hygiene. ${ }^{30}$ Additionally, many people with CF typically utilize cycled inhaled tobramycin as part of their routine treatment regimen in the home. If nebulizer supplies are not obtained from the $\mathrm{CF}$ 
clinic or are not specified in the prescription, different nebulizer types (ie, single-use disposables) may be provided by home care suppliers or pharmacies. Additionally, inefficient aerosol compressors that do not provide the same specifications as ones approved to deliver TIS may be obtained, which means that, even with the approved FDA nebulizer, there could still be efficiency issues if the compressor is inadequate.

Use of less efficient nebulizers may be adequate to deliver other nebulized CF medications, such as albuterol, but it can adversely impact the delivery of concentrationdependent antibiotics such a tobramycin. Clinicians need to be aware of the delivery efficiency of the nebulizer being used to treat patients who require inhaled tobramycin therapy to ensure that optimal concentrations of antibiotic reaches the lung. When these concentrations are not optimally achieved, reduced concentration of antibiotic may have adverse consequences on bacterial persistence and antibiotic resistance, as demonstrated in this standardized controlled in vitro study. Therefore, respiratory therapists should be aware of the significance of the in vitro findings of this study when considering the choice of nebulizer for inhaled tobramycin therapy for individuals with $\mathrm{CF}$.

Several factors can be responsible for exposure of $P$. aeruginosa to sublethal concentrations of tobramycin (Table 1), including patient-related factors that are difficult to control, such as disease severity and individual adherence to nebulized treatments. The experimental approach of the current in vitro study was designed to circumvent the complexities of an in vivo study by simply examining in vitro susceptibility with a single variable, namely tobramycin concentration. Selection of a more efficient nebulizer to deliver higher concentrations of antibiotic to the CF lung is one variable clinicians can control to avoid sublethal concentrations that may encourage development of antimicrobial resistance. To date, there have been no reports, either in vivo or in vitro, that have examined the potential correlation between low concentrations of tobramycin extrapolated from less efficient nebulizers and antimicrobial resistance. Therefore, the main aim of this in vitro study was to examine the effect of exposing clinical isolates of $P$. aeruginosa to sublethal concentrations of TIS to emulate poor nebulizer performance and to identify whether such exposure resulted in altered bacterial persistence and increased antibiotic resistance to tobramycin.

\section{Effect on Bacterial Persistence}

It is important that nebulizers deliver sufficient concentrations of tobramycin that exceed the MIC of newly acquired $P$. aeruginosa isolates. Failure to deliver adequate nebulized concentrations of antibiotic to the CF airways will allow $P$. aeruginosa to persist and thereby evade eradication. This leads to chronic colonization and makes future attempts at eradication much less successful.

\section{Effect on Antimicrobial Resistance}

Antimicrobial resistance is an important problem in managing individuals with $\mathrm{CF}$, leading to reduced options of available antibiotic classes, which may lead to treatment dilemmas. Antimicrobial resistance may manifest in P. aeruginosa at 2 stages, namely on first isolation of the organism and during the chronic phase of infection. It is unusual that first isolates are resistant to antipseudomonal antibiotics, indicating that these isolates originate in the environment, where they are not exposed to such antibiotics and are sensitive to antipseudomonal antibiotics. However, occasionally first isolates may be resistant to certain antibiotics, which suggests acquisition of an organism from other people with $\mathrm{CF}$ or from the health care environment and not as the consequence of a true environmental acquisition.

Once early colonization has been established, there are several clinical scenarios that may allow antimicrobial resistance to develop: the use of antibiotics in oral, nebulized/inhaled, and intravenous formulations; duration and frequency of use of antibiotics; and any clinical scenario that could potentially lead to exposure of the organism to suboptimal concentrations of the antibiotic. These include poor patient compliance in taking antibiotics, poor antimicrobial stewardship practices, and failure to deliver antibiotic at optimal concentration to the lungs due to the use of less efficient nebulizers (Table 1).

When the MIC of $P$. aeruginosa organisms already exceeds the peak TIS concentration delivered by the nebulizer, then tobramycin no longer offers effective bactericidal activity, leading to persistence of $P$. aeruginosa and ineffective clearance/eradication. The clinical importance of such data emphasizes the importance of nebulizer efficiency in maximizing the antibiotic concentrations delivered by the nebulizer, so that the concentrations delivered are greater than the MIC value of the infecting strain, thus optimizing first isolate eradication and suppression of chronic $P$. aeruginosa isolates. When MIC values increase due to the factors highlighted above, then inefficient nebulizers may no longer deliver antibiotic at sufficient concentrations to be bactericidal.

\section{Importance of Cycling Nebulized Tobramycin}

On comparison of MIC values across arms $1-3$, it was noted that the MIC after a $28-\mathrm{d}$ ON $+28 \mathrm{~d}$ OFF cycle of tobramycin was significantly lower than after a single 28$\mathrm{d}$ ON cycle $(P=.008)$ (see the supplementary materials at http://www.rcjournal.com). This is the first in vitro evidence that a 28-d OFF cycle promotes an increase in tobramycin susceptibility, providing evidence in support of the conventional treatment regime of $28 \mathrm{~d} \mathrm{ON}+28 \mathrm{~d}$ OFF. Historically, early tobramycin treatment regimens included a 28-d OFF cycle, with the concept of 
minimizing the development of tobramycin resistance, but early studies did not provide an evidence base that helped prove the need for this OFF period to minimize resistance. Our results substantiate the importance of this OFF cycle for tobramycin. Antibiotic susceptibility was greatest at the end of the 28-d OFF cycle (see the supplementary materials at http://www.rcjournal.com); therefore, it is important to ensure that individual adherence to nebulized tobramycin is maximized when the next $28-\mathrm{d}$ ON cycle commences. The aim is to exploit the vulnerability in MIC values created by the preceding 28-d OFF cycle.

Furthermore, many other treatment regimens employing antipseudomonal nebulized antibiotics other than tobramycin have copied the 28-d OFF period without providing any evidence of what this means for resistance development with that particular antibiotic. Given that many of these other inhaled antibiotics, including colistin, aztreonam, and levofloxacin, belong to other classes of antibiotics (ie, not aminoglycosides), each with their own unique biology, including class-specific mode of action and associated mechanisms of antibiotic resistance, it is unwise to extrapolate the findings of this aminoglycoside to other antibiotics in terms of justification for their 28-d OFF cycle. Therefore, similar studies of other non-aminoglycoside antipseudomonal antibiotics are needed to substantiate the impact of a 28 -d OFF period and what this means for resistance development for these antibiotics.

\section{Importance of Nebulizer Choice}

Previous in vitro comparisons of nebulizers have demonstrated a wide variation in nebulizer delivery efficiency. ${ }^{10}$ In an in vitro study of 20 jet nebulizer/air source combinations (18 disposable and 2 reusable) comparing the delivery efficiency of TIS $(300 \mathrm{mg} / 5 \mathrm{~mL})$, the combination of the nebulizer, interface, and air source can have a significant impact on the respirable dose of TIS to the lungs..$^{10}$ Vecellio et $\mathrm{al}^{10}$ concluded that none of the disposable nebulizers tested could be recommended as an alternative to the Pari LC Plus for tobramycin delivery, due to the lower respirable TIS doses with the studied nebulizer/air source combinations. Vecellio et $\mathrm{al}^{10}$ did not investigate the potential clinical consequences of these performance differences, such as bacterial persistence and antimicrobial resistance, which was the aim of our study.

\section{Study Limitations}

While the adoption of this in vitro study helped circumvent several variables, as discussed above, it also presents several limitations, as does any in vitro study, when attempting to apply the data to an in vivo setting. Because no in vitro or in vivo data exist, a controlled in vitro study offers the opportunity to examine one variable that would be difficult to control in a clinical trial due to multiple confounders. We therefore focused entirely on the in vitro effects of tobramycin concentration on antibiotic susceptibility of $P$. aeruginosa isolates grown in the presence of subinhibitory concentrations of tobramycin. No nebulizers were employed to physically deliver tobramycin to the $P$. aeruginosa isolates; rather isolates were incubated in the presence of extrapolated tobramycin concentrations. Extrapolated concentrations were in turn derived from previously published fine particle mass experimental data, which had varying standard deviations. ${ }^{10}$ Furthermore, organisms were challenged at constant peak concentrations of tobramycin. We know that this is not the case in vivo, as previous pharmacokinetics and pharmacodynamics studies have shown the peak inhaled tobramycin concentration in sputum is achieved with the Pari LC Plus $\sim 30 \mathrm{~min}$ after first delivery and is then reduced to approximately one third ( $~ 750 \mathrm{vs} 250 \mu \mathrm{g} / \mathrm{g}$ sputum) by $1.5 \mathrm{~h}$ later. ${ }^{14}$ However notwithstanding these limitations, our results indicate that concentrations of tobramycin below the inhibitory threshold have the potential to promote a decrease in tobramycin susceptibility. Although this in vitro study does not present any data to indicate that this happens in vivo, this is an important finding, one which clinicians should be aware of given the potential for this happening in vivo.

Data from our study expand upon the results reported by Vecellio et $\mathrm{al}^{10}$ and indicate how extrapolated peak and trough tobramycin concentrations from less efficient nebulizers can be important drivers in vitro of both bacterial persistence and antimicrobial resistance in $P$. aeruginosa organisms in patients with CF. Poor antimicrobial stewardship, poor treatment adherence, and suboptimal pharmacokinetic/pharmacodynamics parameters are well associated with driving antibiotic resistance. This study supports how nebulizer choice can be an important custodian of $P$. aeruginosa eradication as well as antimicrobial stewardship. This is an important clinical consideration to maximize organism eradication and minimize antibiotic resistance development.

\section{Conclusions}

Results of this in vitro study emulating standard cycles of ON/OFF inhaled antipseudomonal tobramycin therapy indicate that suboptimal concentrations of tobramycin drove increased antibiotic resistance. Extrapolated tobramycin trough concentrations derived from less efficient nebulizers drove antibiotic resistance development by initially allowing the survival of intermediate and resistant organisms because nebulizer performance did not achieve critical MIC sufficient to eradicate the organism, and by allowing the development of resistance in those surviving cells. For people with CF on TIS treatment, it is important that clinicians and individuals utilize a nebulizer that helps mitigate the possibility of an upward drift in antibiotic resistance, thereby protecting the clinical value of TIS within CF care. 


\section{Nebulized Tobramycin Concentration and Antimicrobial Resistance}

\section{REFERENCES}

1. Cystic Fibrosis Foundation. About cystic fibrosis. Available at: https:// www.cff.org/What-is-CF/About-Cystic-Fibrosis. Accessed February 17, 2021.

2. Mogayzel PJ Jr, Naureckas ET, Robinson KA, Mueller G, Hadjiliadis D, Hoag JB, et al. Cystic fibrosis pulmonary guidelines: chronic medications for maintenance of lung health. Am J Respir Crit Care Med 2013;187(7):680-689.

3. Smith S, Rowbotham NJ, Regan KH. Inhaled anti-pseudomonal antibiotics for long-term therapy in cystic fibrosis. Cochrane Database Syst Rev 2018;3:CD001021. doi: 10.1002/14651858.CD001021.pub3.

4. Castellani C, Duff AJA, Bell SC, Heijerman HGM, Munck A, Ratjen F, et al. ECFS best practice guidelines: the 2018 revision. J Cyst Fibros 2018;17(2):153-178.

5. Daniels T, Mills N, Whitaker P. Nebuliser systems for drug delivery in cystic fibrosis. Cochrane Database Syst Rev 2013;4:CD007639. doi: 10.1002/14651858.CD007639.pub2.

6. Westerman EM, Boer AH, Touw DJ, Brun PP, Roldaan AC, Frijlink $\mathrm{HW}$, et al. Aerosolization of tobramycin (TOBI) with the Pari LC Plus reusable nebulizer: which compressor to use? Comparison of the CR60 to the PortaNeb compressor. J Aerosol Med Pulm Drug Deliv 2008;21(3):269-280

7. Electronic Medicines Compendium. Tobramycin. 2020. Available at: https://www.medicines.org.uk/emc/search? $\mathrm{q}=$ tobramycin. Accessed September 3, 2020.

8. Daniels T, Goodacre L, Sutton C, Pollard K, Conway S, Peckham D. Accurate assessment of adherence: self-report and clinician report vs electronic monitoring of nebulizers. Chest 2011;140(2):425-432.

9. Conway SP, Pond MN, Hamnett T, Watson A. Compliance with treatment in adult patients with cystic fibrosis. Thorax 1996;51(1):29-33.

10. Vecellio L, Abdelrahim ME, Montharu J, Galle J, Diot P, Dubus JC. Disposable versus reusable jet nebulizers for cystic fibrosis treatment with tobramycin. J Cyst Fibros 2011;10(2):86-92.

11. Clavel A, Boulaméry A, Bosdure E, Luc C, Lanteaume A, Gorincour $\mathrm{G}$, et al. Nebulisers comparison with inhaled tobramycin in young children with cystic fibrosis. J Cyst Fibros 2007;6(2):137-143.

12. Eisenberg J, Pepe M, Pepe M, Vasiliev M, Montgomery AB, Smith AL, Ramsey BW. A comparison of peak sputum tobramycin concentration in patients with cystic fibrosis using jet and ultrasonic nebulizer systems. Chest 1997;111(4):955-962.

13. Geller DE, Rosenfeld M, Waltz DA, Wilmott RW, AeroDose TOBI Study Group. Efficiency of pulmonary administration of tobramycin solution for inhalation in cystic fibrosis using an improved drug delivery system. Chest 2003;123(1):28-36.

14. Hubert D, Leroy S, Nove-Josserand R, Murris-Espin M, Mely L, Dominique $\mathrm{S}$, et al. Pharmacokinetics and safety of tobramycin administered by the Pari eFlow rapid nebulizer in cystic fibrosis. J Cyst Fibros 2009;8(5):332-337.

15. Lenney W, Edenborough F, Kho P, Kovarik JM. Lung deposition of inhaled tobramycin with eFlow rapid/LC Plus jet nebuliser in healthy and cystic fibrosis subjects. J Cyst Fibros 2011;10(1):9-14.
16. Ramsey BW, Dorkin HL, Eisenberg JD, Gibson RL, Harwood IR, Kravitz RM, et al. Efficacy of aerosolized tobramycin in patients with cystic fibrosis. N Engl J Med 1993;328(24):1740-1746.

17. Food and Drug Administration. Approved Drug Products with Therapeutic Equivalence Evaluations (Orange Book), 40th edition. 2020. Available at: https://www.fda.gov/media/71474/download. Accessed September 3, 2020

18. Cystic Fibrosis Foundation Patient Registry 2018 Annual Data Report. Available at: https://www.cff.org/Research/ResearcherResources/Patient-Registry/2018-Patient-Registry-Annual-DataReport.pdf. Accessed September 3, 2020.

19. Lester MK, Eidson DM, Cystic Fibrosis Foundation Nebulizer and Compressor Task Force. Acquisition and use of nebulizer and compressor equipment in cystic fibrosis: a national survey of patients and providers. Ped Pulmonol 2019;54(S2):S478.

20. Kidd TJ, Canton R, Ekkelenkamp M, Johansen HK, Gilligan P, LiPuma JJ, et al. Defining antimicrobial resistance in cystic fibrosis. J Cyst Fibros 2018;17(6):696-704.

21. McCarthy K. Pseudomonas aeruginosa: evolution of antimicrobial resistance and implications for therapy. Semin Respir Crit Care Med 2015;36(1):44-55.

22. Gramegna A, Moore JE, McCaughan J, Millar BC, Ewing J, Elborn JS, et al. Increasing burden of antimicrobial resistance in Pseudomonas aeruginosa from adult patients with cystic fibrosis (CF) in Northern Ireland: then and now. Ulster Med J 2018;87(2):129-130.

23. Smyth AR, Bell SC, Bojcin S, Bryon M, Duff A, Flume P, et al. European Cystic Fibrosis Society standards of care: best practice guidelines. J Cyst Fibros 2014;13(Suppl 1):S23-S42.

24. Döring G, Flume P, Heijerman H, Elborn JS, Consensus Study Group. Treatment of lung infection in patients with cystic fibrosis: current and future strategies. J Cyst Fibros 2012;11(6):461-479.

25. Flume PA, VanDevanter DR. Clinical applications of pulmonary delivery of antibiotics. Adv Drug Deliv Rev 2015;85:1-6.

26. Mustafa MH, Chalhoub H, Denis O, Deplano A, Vergison A, Rodriguez-Villalobos $\mathrm{H}$, et al. Antimicrobial susceptibility of Pseudomonas aeruginosa isolated from cystic fibrosis patients in Northern Europe. Antimicrob Agents Chemother 2016;60(11):6735-6741.

27. Raymond B. Five rules for resistance management in the antibiotic apocalypse, a road map for integrated microbial management. Evol Appl 2019;12(6):1079-1091.

28. Clinical and Laboratory Standards Institute (CLSI). Performance Standards for Antimicrobial Susceptibility Testing. 29th ed. Wayne, PA: Clinical and Laboratory Standards Institute; 2019.

29. Marx A, Backes C, Meese E, Lenhof HP, Keller A. EDISON-WMW: Exact dynamic programing solution of the Wilcoxon-Mann-Whitney test. Genomics Proteomics Bioinformatics 2016;14(1):55-61.

30. Weber DJ, Gergen MF, Sickbert-Bennett EE, Short KA, LanzaKaduce KE, Rutala WA. Frequency of contamination of singlepatient-use nebulizers over time. Infect Control Hosp Epidemiol 2014;35(12):1543-1546 